\section{OPEN ACCESS}

Edited by:

Petra Zimmermann,

Université de Fribourg, Switzerland

Reviewed by:

Rinawati Rohsiswatmo,

RSUPN Dr. Cipto

Mangunkusumo, Indonesia

Hao Chen,

Huazhong University of Science and

Technology, China

*Correspondence:

Massimo Luca Castellazzi

luca.castellazzi@policlinico.mi.it

Specialty section

This article was submitted to Pediatric Infectious Diseases,

a section of the journal

Frontiers in Pediatrics

Received: 10 April 2021

Accepted: 12 July 2021

Published: 04 August 2021

Citation:

Castellazzi ML, Corsello A, Cerrato L,

Carnevali A, Morandi A, Leva E,

Agostoni CV and Marchisio $P$ (2021)

Intussusception in an Infant With

SARS-CoV-2 Infection: A Case Report

and a Review of the Literature.

Front. Pediatr. 9:693348.

doi: $10.3389 /$ fped.2021.693348

\title{
Intussusception in an Infant With SARS-CoV-2 Infection: A Case Report and a Review of the Literature
}

\begin{abstract}
Massimo Luca Castellazzi ${ }^{1 *}$, Antonio Corsello ${ }^{2}$, Lucia Cerrato ${ }^{2}$, Alessandra Carnevali ${ }^{3}$, Anna Morandi ${ }^{4}$, Ernesto Leva ${ }^{4}$, Carlo Virginio Agostoni ${ }^{5,6}$ and Paola Marchisio ${ }^{7,8}$

${ }^{1}$ Fondazione IRCCS Ca' Granda Ospedale Maggiore Policlinico, Pediatric Emergency Department, Milan, Italy, ${ }^{2}$ University of Milan, Milan, Italy, ${ }^{3}$ Fondazione IRCCS Ca' Granda Ospedale Maggiore Policlinico, Radiology Unit - Pediatric Division, Milan, Italy, ' ${ }^{4}$ Fondazione IRCCS Ca' Granda Ospedale Maggiore Policlinico, Pediatric Surgery, Milan, Italy, ' Fondazione IRCCS Ca' Granda Ospedale Maggiore Policlinico, Pediatric Intermediate Care Unit, Milan, Italy, ${ }^{6}$ Department of Clinical Sciences and Community Health, University of Milan, Milan, Italy, ${ }^{7}$ Fondazione IRCCS Ca' Granda Ospedale Maggiore Policlinico, Pediatric Highly Intensive Care Unit, Milan, Italy, ${ }^{8}$ Department of Pathophysiology and Transplantation, University of Milan, Milan, Italy
\end{abstract}

Coronavirus disease 2019 (COVID-19) is caused by acute respiratory syndrome coronavirus 2 (SARS-CoV-2). Even if predominantly considered a respiratory pathogen, it could be associated with gastrointestinal involvement, generally in mild forms. Recent reports highlight the association between SARS-CoV-2 and intussusception in infants. A case of intussusception is hereby described in a previously healthy infant in whom the diagnosis of SARS-CoV-2 was made after the analysis of bronchoalveolar lavage and intraoperative specimens following surgical procedures. Accordingly, a review of infant cases with intussusception and SARS-CoV-2 infection is also reported.

Keywords: intussusception, SARS-CoV-2, COVID-19, gastrointestinal symptoms, infants

\section{INTRODUCTION}

Intussusception, defined as the invagination of a proximal segment of the intestine into an adjacent distal segment, is considered a common cause of intestinal obstruction in patients with acute abdominal pain, especially in infants within 10 months of age. The most frequent form in pediatric age is the ileocolic (80\%), followed by rare ileoileal and colocolic forms (1). Viral infections have been described as associated with this condition (2).

The severe acute respiratory syndrome-coronavirus-2 (SARS-CoV-2) was firstly identified in patients with a severe form of pneumonia in Wuhan in December 2019 and subsequently became pandemic (3).

The coronavirus disease 2019 (COVID-19) has been associated with a wide range of clinical presentations, from asymptomatic or mild respiratory symptoms to severe lung injury, multi-organ failure and death. However, it is now evident that SARS-CoV-2 can also affect the gastrointestinal system, with the highest incidence in pediatric age (4). Sporadic cases of intussusception in SARS-CoV-2 infected children have been reported worldwide (2).

Here, we report a case of a female infant with ileocolic invagination whose diagnosis of SARS$\mathrm{CoV}-2$ infection was achieved after an extended diagnostic workout. Furthermore, a literature review on this topic is also performed considering clinical presentation, diagnosis, treatment options and outcome. 


\section{CASE PRESENTATION}

A previously healthy 10-month-old girl was admitted to our emergency department for abdominal pain, accompanied by irritability, decreased oral intake, intermittent crying and two episodes of non-biliary vomiting during the previous $24 \mathrm{~h}$. No fever or presence of bloody stools were referred in previous days.

On physical examination, she presented apyretic with good general conditions and normal vital signs. An abdominal distension was clearly observable, with crying and pain evoked by the palpation of the left lower quadrant. No signs of dehydration or respiratory distress were noted. A rectal probe was applied, which showed the presence of blood traces. Laboratory tests performed on admission showed a normal white blood cell count and a mild increase of C-reactive protein of $10.2 \mathrm{mg} / \mathrm{L}$ (normal value $<5 \mathrm{mg} / \mathrm{L}$ ). Renal, liver function tests and electrolytes resulted normal as well. Blood gas analysis was normal. A coproculture and research for adenovirus and rotavirus on stool sample were performed and subsequently resulted negative. Urine analysis did not show signs of urinary tract infection.

Due to patient's age and clinical presentation, an abdominal ultrasound was performed, showing the presence of a massive ileocolic intussusception, with increased thickness of the intestinal wall and accompanying mesentery, and enlarged lymph nodes (Figure 1A). A subsequent contrast enema, using Gastrografin ${ }^{\circledR}$ (diatrizoate meglumine and diatrizoate sodium solution) diluted with saline, was successfully performed reducing the invagination (Figure 1B). No complications occurred during the procedure.

No history or signs of SARS-CoV-2 infection was documented in the patient and in close relatives, but, due to the pandemic period, the patient and her mother were tested for SARS-CoV2 with polymerase chain reaction (PCR) molecular testing on nasopharyngeal aspirate and swab, respectively. The mother resulted positive for SARS-CoV-2, while the patient resulted negative. A subsequent nasopharyngeal aspirate performed $24 \mathrm{~h}$ after the first test was performed and it confirmed the negativity for SARS-CoV-2. The patient, admitted to the ward, underwent intravenous antibiotic treatment with ceftriaxone $(100 \mathrm{mg} / \mathrm{kg} /$ day $)$ and metronidazole $(20 \mathrm{mg} / \mathrm{kg} /$ day in 3 doses $)$ until discharge.

An intravenous fluid replacement was administered for 2 days and then progressively reduced and interrupted on day 4 following adequate oral intake and tolerance. During the first 4 days of hospitalization, the patient experienced recurrent episodes of spiking colicky pain with spontaneous resolution in few minutes, and on day 5 an abdominal ultrasound evidenced a recurrence of the ileocolic intussusception at the level of the splenic flexure. A new non-surgical hydrostatic reduction procedure was attempted but several relapses occurred during this procedure (Figure 2). A surgical reduction was therefore indicated. Before performing the procedure, a further SARSCoV-2 PCR molecular testing on nasopharyngeal aspirate was then obtained, with negative result. Laparotomy with manual reduction of the ileocolic invagination and ileocecopexy was subsequently performed. Macroscopically, numerous enlarged abdominal lymph nodes, especially among celiac and mesenteric ones, near the ileocecal junction, were found. Due to the COVID-19 exposure, and the persistent negativity of SARSCoV-2 PCR molecular testing on nasopharyngeal aspirate, a molecular test was performed on a bronchoalveolar lavage (BAL) sample, a parietal peritoneum biopsy and a stool sample obtained during surgery, respectively. Positivity for SARS-CoV2 was detected on BAL and biopsy, while stools were negative. The postoperative course was uneventful and the patient was discharged after a total of 11 days of hospitalization. A timeline of clinical course, laboratory test for SARS-CoV-2, radiological investigations and treatment of intussusception is summarized in Figure 3. During the hospital course, the patient did not develop any respiratory symptoms or signs of SARS-CoV-2 infection other than the intussusception. No further nasopharyngeal aspirate was performed for SARS-CoV-2 as the patient was completely asymptomatic.

\section{DISCUSSION}

Since the emergence of COVID-19 pandemic, it has been reported that children, and particularly infants, may have an asymptomatic or mild clinical presentation of the infection. Gastrointestinal symptoms, including nausea, vomiting, diarrhea, and abdominal pain have been frequently reported in the pediatric age (4). The angiotensin-converting enzyme 2 receptor (ACE-2) and transmembrane protease, serine 2 (TMPRSS2), co-expression in the same cell are essential for the entry of the virus (5). These two proteins can be expressed at a high level in the glandular cells of the gastric, duodenal, and rectal epithelium, and the enterocytes of the ileum and colon (6), so giving biologic plausibility to the gastrointestinal involvement in SARS-CoV-2 infection.

Intussusception is the leading cause of gastrointestinal obstruction in infancy. Once diagnosed, a prompt reduction of the intussusception should be performed due to the possible risk of bowel ischemia and necrosis, bowel perforation and peritonitis (7). A non-surgical reduction through pneumatic or hydrostatic enema is suggested as first line approach in the pediatric age, with a success rate close to $90 \%$ (8). Nearly $90 \%$ of intussusceptions are considered idiopathic, mainly linked to findings of lymphoid hyperplasia of the small intestine directly promoting the invagination (1). However, there is a very strong casual association between viruses, mesenteric lymphoid hyperplasia and intussusception (9). In particular, adenovirus, enterovirus, norovirus, rotavirus and human herpes virus 6 have been associated with intussusception in infants and young children (10). To the best of our knowledge, eight cases of intussusception being the presentation of COVID-19 in infants have been reported so far (11-17). The main findings from these reports are summarized in Supplementary Table 1.

The age of presentation ranges from 3 to 10 months with a male prevalence (five out of eight infants). Most reports describe an irritable baby with abdominal pain and jelly-like stools. Fever was documented in 5 cases $(11,12,14,15)$. A concurrent or a recent history of respiratory tract infection was described in only three cases (11-13). Six of the eight patients were diagnosed as 

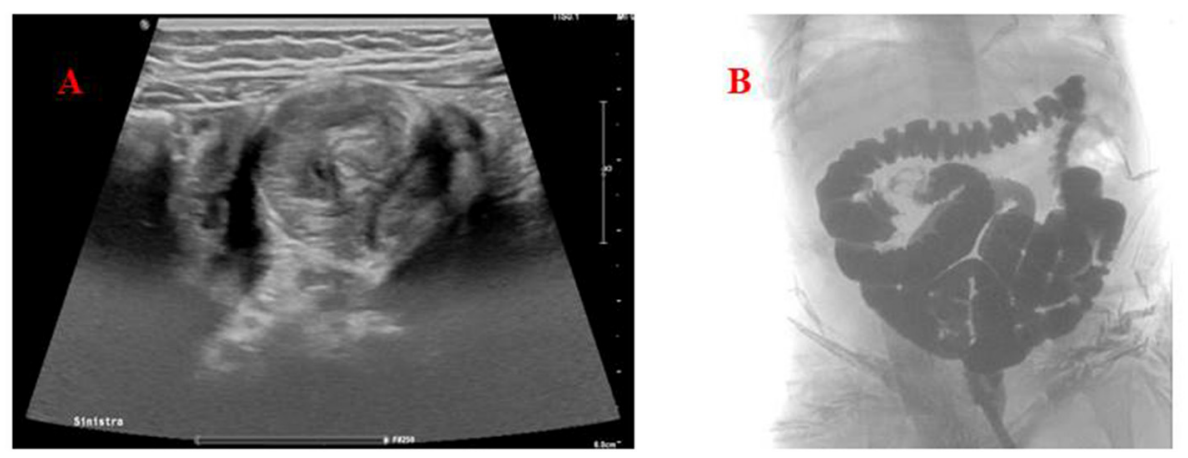

FIGURE 1 | (A) Abdomen ultrasound. The target (donut) sign found in the left lower quadrant, confirming the diagnosis of intussusception. (B) Hydrostatic reduction of intussusception using Gastrografin ${ }^{\circledR}$ contrast solution showing the successful reduction of the ileocolic invagination.
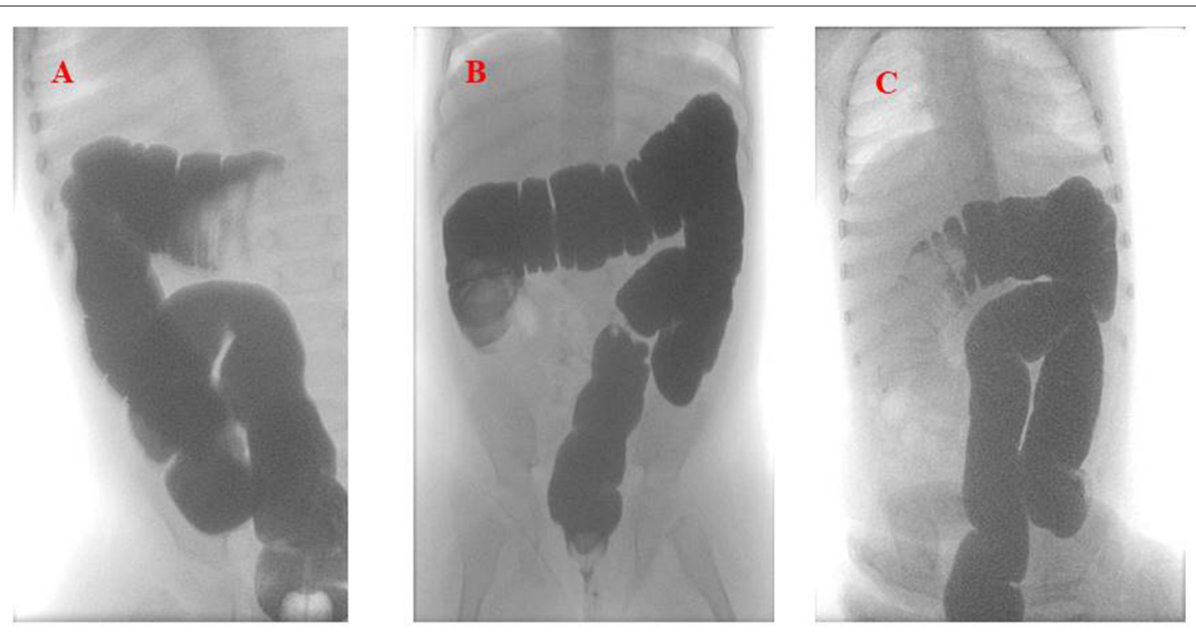

FIGURE 2 | Hydrostatic reduction of intussusception using Gastrografin ${ }^{\circledR}$ contrast solution (A). The first imaging shows the recurrence of the intussusception in the splenic flexure. (B,C) Show the relapses of the intussusception in the ascending and then back in the transverse colon, respectively.

ileocolic intussusception, one as ileocecal intussusception and in the last case the kind of invagination was not clarified. Surgical procedure was performed in four cases, two of which preceded by an unsuccessful pneumatic reduction $(12,15,17)$. Only one case reported the patient's death due to the development of a multiorgan dysfunction syndrome (15), whereas the remaining cases were all discharged without sequelae $(11-14,16,17)$.

For the direct identification of SARS-CoV-2, the PCR molecular testing should be performed on specimens taken from the upper (nasopharyngeal swab or aspirate and oropharyngeal swab) and the lower respiratory tract (BAL, tracheal aspirate and sputum) $(18,19)$. Since higher viral loads are usually detected in the nose compared to the throat, the World Health Organization recommends to prioritize nasopharyngeal swab over oropharyngeal swab $(19,20)$. All the described infants had a diagnosis of SARS-CoV-2 infection through a PCR molecular testing but only few authors reported the type of sample used for the test, in particular whether nasal or throat swabs $(13,15,17)$. Furthermore, the majority of the cases (5) had no previous direct exposure to COVID-19 $(12,13,15,16)$.
Accordingly, the hereby reported 10-month old patient presented with an ileocolic intussusception, confirmed at abdomen ultrasound, with the classical presentation of abdominal pain, vomiting, lack of appetite and blood in stools. Of note, she was apyretic, and neither previous or current respiratory symptoms, nor direct exposure to COVID-19 infected patients were reported in either the patient and her close relatives. Following our Institutional norms, a PCR testing was performed on nasopharyngeal aspirate and swab from the patient and her mother, respectively, and only the mother resulted positive for SARS-CoV-2. In occasion of the surgical manual reduction following the relapse of symptoms, a PCR molecular testing on BAL and biopsy specimens were performed and both resulted positive for SARS-CoV-2. To the best of our knowledge, this is the first report in which SARS-CoV-2 was detected in these specimens in infants with intussusceptions. Accordingly, we may suggest to expand the diagnostic workout in cases where the nasal swab of the patient with intestinal intussusception is negative, but there is strong evidence of recent direct exposure to SARS-CoV-2, up to specific gut bioptic 


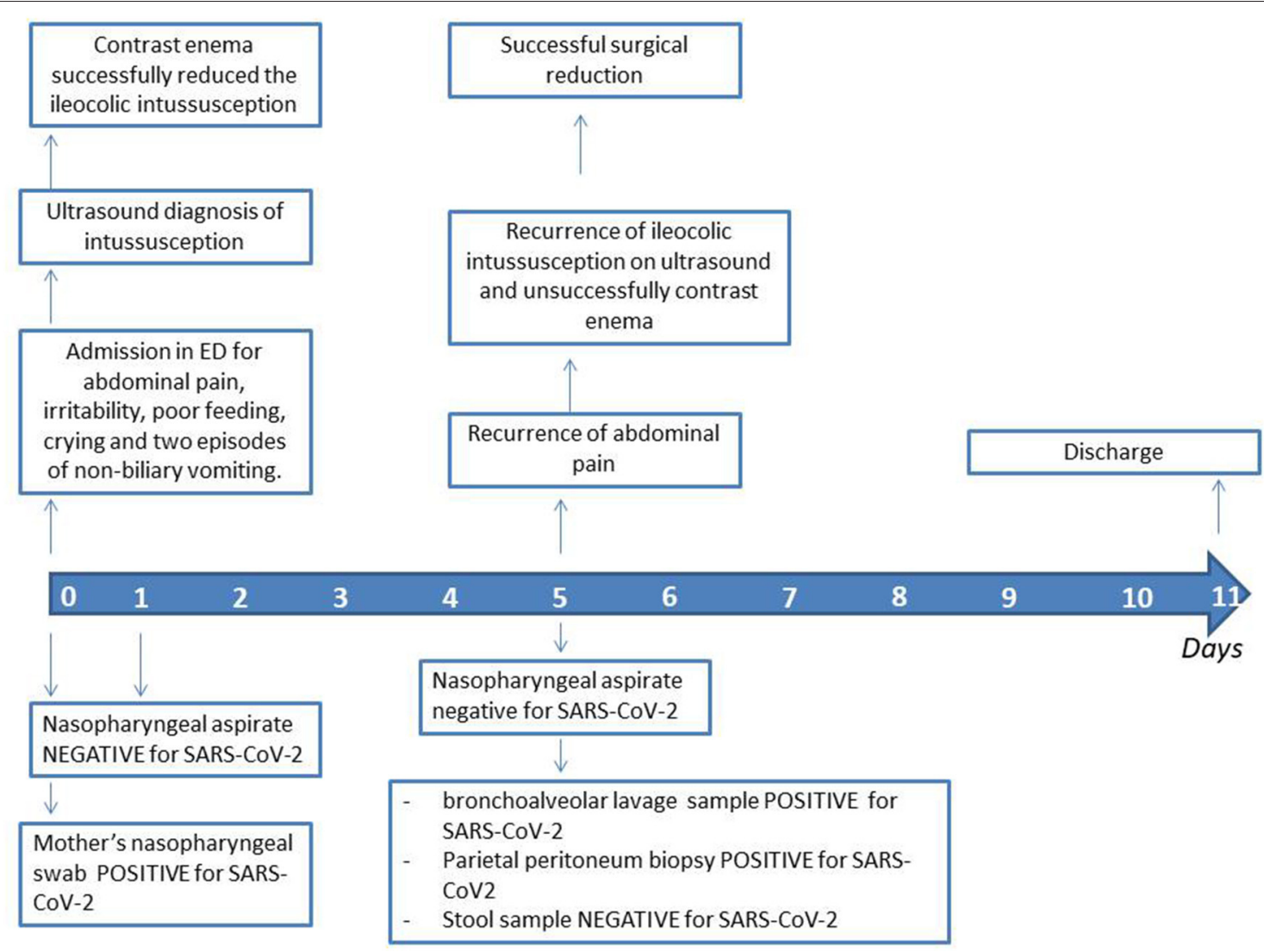

FIGURE 3 | Timeline course of the patient.

specimen, when feasible due to intercurring surgical procedures, as in our case. Without the clear positivity to SARS-CoV-2 observed in the mother, no further tests would have probably been conducted on this patient, with a possible underestimation of the diagnosis. Almost unexpectedly, the stools specimens of our patient resulted negative for SARS-CoV-2 even if 8 out of 10 COVID-19 positive children have been reported with positive rectal swabs, in spite of negative nasopharyngeal swabs (21). Accordingly, although viral shedding in COVID-19 patients primarily occurs through nasal secretions, a feco-oral route of transmission has also been postulated.

The mechanism linking the development of intussusception in pediatric patients with SARS-COV-2 infection is not fully understood (16). Previous reports have suggested an association between viral infections, whichever the nature, and a delay in gut peristaltic movements, finally favoring a spontaneous gliding of adjacent bowel loops toward each other (22).

\section{CONCLUSION}

As described, COVID-19 may be implicated in the development of intussusception in infants, even in absence of a systemic and respiratory involvement. Due to the possible link with infectious pathogens, testing for viral pathogens, including SARS-CoV-2, should be performed in infants with symptoms consistent with intussusception. In those infants with a history of direct SARS-CoV-2 exposure, we hereby suggest to enlarge the diagnostic workout for SARS-CoV-2 to feasible samples in case nasopharyngeal swab or aspirate result negative, in order to achieve a precise diagnosis, whether positive or negative.

\section{DATA AVAILABILITY STATEMENT}

The original contributions presented in the study are included in the article/Supplementary Materials, further inquiries can be directed to the corresponding author.

\section{ETHICS STATEMENT}

Ethical approval was not provided for this study on human participants because it is a case report. Written informed consent to participate in this study was provided by the participants' legal guardian/next of kin. 


\section{AUTHOR CONTRIBUTIONS}

MC, ACo, and LC designed the topic and wrote the first draft of the manuscript. MC, PM, and CA contributed to the clinical diagnosis. $\mathrm{AM}$ and $\mathrm{EL}$ contributed to patient management. ACa performed the radiological studies. $\mathrm{PM}, \mathrm{CA}, \mathrm{AM}$, and $\mathrm{EL}$ revised the manuscript. All authors read and approved the final manuscript and agree to be accountable for the content of the work.

\section{REFERENCES}

1. Marsicovetere P, Ivatury SJ, White B, Holubar SD. Intestinal intussusception: etiology, diagnosis, and treatment. Clin Colon Rectal Surg. (2017) 30:309. doi: 10.1055/s-0036-1593429

2. Giovanni JE, Hrapcak S, Melgar M, Godfred-Cato S. Global reports of intussusception in infants with SARS-CoV-2 infection. Pediatr Infect Dis J. (2021) 40:e35-6. doi: 10.1097/INF.0000000000002946

3. Zhu N, Zhang D, Wang W, Li X, Yang B, Song J, et al. A novel coronavirus from patients with pneumonia in China, 2019. N Engl J Med. (2020) 382:72733. doi: 10.1056/NEJMoa2001017

4. Puoti MG, Rybak A, Kiparissi F, Gaynor E, Borrelli O. SARS-CoV2 and the gastrointestinal tract in children. Front Pediatr. (2021) 9:617980. doi: 10.3389/fped.2021.617980

5. Wong SH, Lui RN, Sung JJ. Covid-19 and the digestive system. J Gastroenterol Hepatol. (2020) 35:744-8. doi: 10.1111/jgh.15047

6. Chiappini E, Licari A, Motisi MA, Manti S, Marseglia GL, Galli L, et al. Gastrointestinal involvement in children with SARS-COV-2 infection: an overview for the pediatrician. Pediatr Allergy Immunol. (2020) 31:925. doi: $10.1111 /$ pai. 13373

7. Gluckman S, Karpelowsky J, Webster AC, McGee RG. Management for intussusception in children. Cochrane Database Syst Rev. (2017) 6:CD006476. doi: 10.1002/14651858.CD006476.pub3

8. Kelley-Quon LI, Arthur LG, Williams RF, Goldin AB, St. Peter SD, Beres AL, et al. Management of intussusception in children: a systematic review. J Pediatr Surg. (2021) 56:587-96. doi: 10.1016/j.jpedsurg.2020.09.055

9. Okimoto S, Hyodo S, Yamamoto M, Nakamura K, Kobayashi M. Association of viral isolates from stool samples with intussusception in children. Int J Infect Dis. (2011) 15:e641-5. doi: 10.1016/j.ijid.2011.05.008

10. Burnett E, Kabir F, Van Trang N, Rayamajhi A, Satter SM, Liu J, et al. Infectious etiologies of intussusception among children $<2$ years old in 4 Asian countries. J Infect Dis. (2020) 221:1499-505. doi: 10.1093/infdis/jiz621

11. Bazuaye-Ekwuyasi EA, Camacho AC, Saenz Rios F, Torck A, Choi WJ, Aigbivbalu EE, et al. Intussusception in a child with COVID-19 in the USA. Emerg Radiol. (2020) 27:761-4. doi: 10.1007/s10140-020-01860-8

12. Mercado-Martínez I, Arreaga-Gutiérrez FJ, Pedraza-Peña AN. Intussusception and SARS-CoV-2 infection. J Pediatr Surg Case Rep. (2021) 67:101808. doi: 10.1016/j.epsc.2021.101808

13. Moazzam Z, Salim A, Ashraf A, Jehan F, Arshad M. Intussusception in an infant as a manifestation of COVID-19. J Pediatr Surg Case Rep. (2020) 59:101533. doi: 10.1016/j.epsc.2020.101533

14. Athamnah MN, Masade S, Hamdallah $\mathrm{H}$, Banikhaled $\mathrm{N}$, Shatnawi W, Elmughrabi $M$, et al. COVID-19 presenting as intussusception in infants: a case report with literature review. J Pediatr Surg Case Rep. (2021) 66:101779. doi: 10.1016/j.epsc.2021.1 01779

\section{FUNDING}

This work was partially funded by a grant of the Italian Ministry of Health (Ricerca Corrente 2021).

\section{SUPPLEMENTARY MATERIAL}

The Supplementary Material for this article can be found online at: https://www.frontiersin.org/articles/10.3389/fped. 2021.693348/full\#supplementary-material

15. Cai X, Ma Y, Li S, Chen Y, Rong Z, Li W. Clinical characteristics of 5 COVID 19 cases with non-respiratory symptoms as the first manifestation in children. Front Pediatr. (2020) 8:258. doi: 10.3389/fped.2020.00258

16. Martínez-Castaño I, Calabuig-Barbero E, Gonzálvez-Piñera J, López-Ayala JM. COVID-19 infection is a diagnostic challenge in infants with ileocecal intussusception. Pediatr Emerg Care. (2020) 36:e368. doi: 10.1097/PEC.0000000000002155

17. Makrinioti H, MacDonald A, Lu X, Wallace S, Jobson M, Zhang F, et al. Intussusception in 2 Children With Severe Acute Respiratory Syndrome Coronavirus-2 Infection. J Pediatric Infect Dis Soc. (2020) 9:5046. doi: 10.1093/jpids/piaa096

18. Wang W, Xu Y, Gao R, Lu R, Han K, Wu G, et al. Detection of SARSCoV-2 in different types of clinical specimens. JAMA. (2020) 323:18434. doi: 10.1001/jama.2020.3786

19. Di Pietro GM, Capecchi E, Luconi E, Lunghi G, Bosis S, Bertolozzi G, et al. Diagnosis of SARS-CoV-2 in children: accuracy of nasopharyngeal swab compared to nasopharyngeal aspirate. Eur J Clin Microbiol Infect Dis. (2021) 7:1-6. doi: 10.1007/s10096-020-04131-z

20. Zou L, Ruan F, Huang M, Liang L, Huang H, Hong Z, et al. SARS-CoV-2 viral load in upper respiratory specimens of infected patients. N Engl J Med. (2020) 382:1177-9. doi: 10.1056/NEJMc2001737

21. Xu Y, Li X, Zhu B, Liang H, Fang C, Gong Y, et al. Characteristics of pediatric SARS-CoV-2 infection and potential evidence for persistent fecal viral shedding. Nat Med. (2020) 26:502-5. doi: 10.1038/s41591-0200817-4

22. Cera SM. Intestinal intussusception. Clin Colon Rectal Surg. (2008) 21:10613. doi: $10.1055 / \mathrm{s}-2008-1075859$

Conflict of Interest: The authors declare that the research was conducted in the absence of any commercial or financial relationships that could be construed as a potential conflict of interest.

Publisher's Note: All claims expressed in this article are solely those of the authors and do not necessarily represent those of their affiliated organizations, or those of the publisher, the editors and the reviewers. Any product that may be evaluated in this article, or claim that may be made by its manufacturer, is not guaranteed or endorsed by the publisher.

Copyright (C) 2021 Castellazzi, Corsello, Cerrato, Carnevali, Morandi, Leva, Agostoni and Marchisio. This is an open-access article distributed under the terms of the Creative Commons Attribution License (CC BY). The use, distribution or reproduction in other forums is permitted, provided the original author(s) and the copyright owner(s) are credited and that the original publication in this journal is cited, in accordance with accepted academic practice. No use, distribution or reproduction is permitted which does not comply with these terms. 